Case Report

\title{
Persistent Pulmonary Atelectasis in Patient with Rheumatoid Arthritis Treated with High-Flow Therapy and High-Frequency Chest Wall Oscillation
}

\author{
Anna Annunziata, Elena Sciarrillo, Maurizia Lanza, Giuseppe Fiorentino \\ Division of Respiratory Physiopathology, Monaldi Hospital, Naples, Italy \\ Email address: \\ anna.annunziata@gmail.com (A. Annunziata)
}

\section{To cite this article:}

Anna Annunziata, Elena Sciarrillo, Maurizia Lanza, Giuseppe Fiorentino. Persistent Pulmonary Atelectasis in Patient with Rheumatoid Arthritis Treated with High-Flow Therapy and High-Frequency Chest Wall Oscillation. American Journal of Internal Medicine. Vol. 7, No. 1, 2019, pp. 9-11. doi: 10.11648/j.ajim.20190701.13

Received: November 14, 2018; Accepted: January 31, 2019; Published: February 22, 2019

\begin{abstract}
Rheumatoid arthritis is a systemic inflammatory disorder that affects $\sim 1-2 \%$ of the population. Although a progressive destruction of cartilage and bone is the main presentation, extra-articular manifestations can occur in about $40 \%$ of patients during the course of their disease and are associated with significant morbidity and increased mortality. Pulmonary complications can frequently occur in these patients, although not always clinically recognized. We present a case of 60 -year-old woman suffering from rheumatoid arthritis came to our department for the presence of exertional dyspnea, cough with poor mucous expectoration, chest pain and worsening of daytime asthenia. At a chest x-ray, evidence of right basal pulmonary atelectasis, for which she had practiced antibiotic therapy with persistence of symptomatology and pulmonary atelectasis. Chest CT showed parenchymal consolidation. The patient was treated with antibiotics and rehabilitation therapy, bronchial endoscopy, with persistence of pulmonary atelectasis. She started nasal high-flow therapy but in the seventh day appears epistaxis. For this reason, she suspended treatment with NHF. At the next rehabilitation session, for the recurrence of epistaxis, an otorhinolaryngology visit was performed that confirms the presence of nasal varices. Therefore, the patient started high-flow therapy with a mouthpiece with the use of High Frequency Chest Wall Oscillation. At the end of rehabilitative treatment we observed clinical and radiological improvement.
\end{abstract}

Keywords: Atelectasis, Rheumatoid Arthritis, Pulmonary Rehabilitation, Nasal High-Flow Therapy (NHF)

\section{Introduction}

It is well known that pulmonary manifestations are frequently associated with rheumatoid arthritis. Some authors emphasize on airway disease being one of the major risk factors of infection in rheumatoid arthritis patients and demonstrate that an obstruction of the peripheral airways could ultimately lead to respiratory failure [1]. Atelectasis is the collapse of lung tissue with loss of volume. The trigger may sometimes remain unknown. The etiopathogenesis is commonly associated with a reduction of effective cough, mucus hypersecretion, poor hydration and a mucus plug that favors the stagnation of dense secretions. Treatment includes maintenance of effective cough, respiratory rehabilitation and removal of the cause when possible.

\section{Case Presentation}

A 60-year-old woman suffering from rheumatoid arthritis came to our department for the presence of exertional dyspnea, cough with poor mucous expectoration, chest pain and worsening of daytime asthenia. Chest $\mathrm{x}$-ray showed evidence of right basal pulmonary atelectasis, for which she had practiced antibiotic therapy with levofloxacin, piperacillin / tazobactam and amikacin with persistence of symptomatology and pulmonary atelectasis.

The patient was treated with sodium methotrexate for rheumatoid arthritis; this therapy had been suspended for about three months, since the onset of pulmonary disease. The last chest CT showed "atelectasis of posterior-basal lobe with 
a slight thickening of the contiguous pleural plan associated with a bronchial ectasias of basal traction and some mediastinal adenopathies maximum $1 \mathrm{~cm}$ ". (Figure 1)

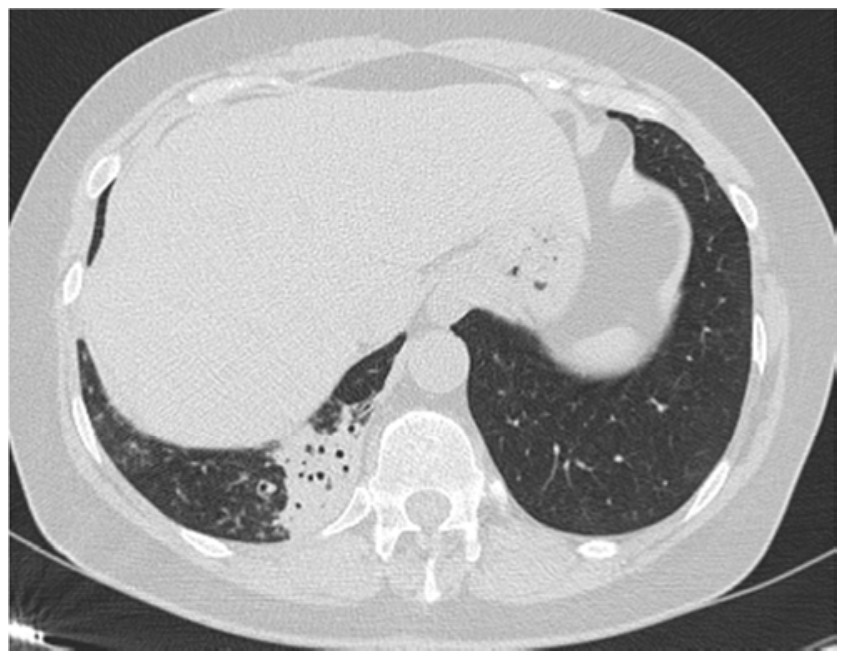

Figure 1. Particular CT: basal atelectasis.

The patient had weight loss of about $2 \mathrm{~kg}$ last month. The spirometry showed a Forced Vital Capacity (FVC) of 2,53 lt (100\% of theoretical), Forced Expiratory Volume in one second (FEV1) 2,15 lt ( $100 \%$ ), DLCOmean 21,68 $\mathrm{ml} / \mathrm{min} / \mathrm{mmHg}(100 \%)$. The peak cough flow was $240 \mathrm{~L} / \mathrm{min}$. Peripheral O2 saturation was $92 \%$. Arterial blood gas analysis showed normocapnia with slight hypoxemia and acid-base balance was normal. Blood cultures were negative. Bronchoscopy showed bronchial normopervietà with hyperemic mucosa and presence of abundant mucous secretions; cytological was negative for malignant cells; the microbiological examination of sputum showed absence of microorganisms; The search for Koch's bacillus was negative, alveolar bronchial lavage showed increase in the lymphocytic and granulocytic neutrophil share with normal CD4 / CD8 ratio.

The patient starts a pulmonary rehabilitation program. For fifty minutes nasal high-flow therapy (NHF) is applied at 45 $\mathrm{L} / \mathrm{min}$ with a $\mathrm{FiO} 2$ of 0.21 , delivering low-level positive airway pressure as respiratory assistance. NHF therapy delivered by the Airvo system is a simple device that supplies heated and humidified air and if necessary blended with oxygen, at a high flow rate via a large-bore nasal cannula. The supplied gases contain a saturated water content of $44 \mathrm{mg}$ $\mathrm{H} 2 \mathrm{O} / \mathrm{L}$ at $37^{\circ} \mathrm{C}$; the humidity is optimal for maintenance of mucociliary function in the airways. Furthermore, we apply for 30 minute day, High-Frequency Chest Wall Oscillation (HFCWO) at pressure of $5 \mathrm{~cm} \mathrm{H} 2 \mathrm{O}$ and a frequency of $13 \mathrm{~Hz}$. We apply also continuous positive airway pressure in left lateral decubitus at $13 \mathrm{~cm} \mathrm{H} 2 \mathrm{O}$ pressure for a time of 30 minute. The treatment was well tolerated.

Unfortunately, in the seventh day appears epistaxis. For this reason, she suspended treatment with NHF. At the next rehabilitation session, for the recurrence of epistaxis, an otorhinolaryngology visit is performed that confirms the presence of nasal varices. Therefore, the patient starts high-flow therapy with a mouthpiece (Figure 2). After four weeks of treatment a complete regression of the symptoms and an improvement of lung function were obtained. Last spirometry performed after about 30 days of treatment showed a variation of Forced Vital Capacity (FVC) of 2,80 L (110\% of theoretical), Forced expiratory volume in one second (FEV1) $2,57 \mathrm{~L}(120 \%)$.

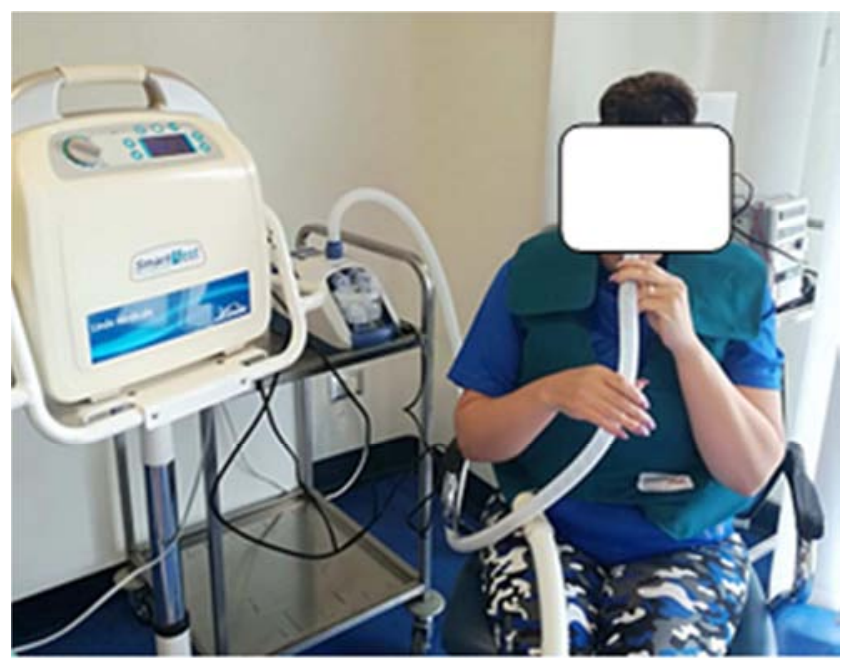

Figure 2. High-flow therapy with a mouthpiece and High-Frequency Chest Wall Oscillation.

Peripheral $\mathrm{O}_{2}$ saturation was $96 \%$. The peak cough flow was $260 \mathrm{~L} / \mathrm{min}$. The chest CT scan shows complete resolution of the atelectasis area. (Figure 3)

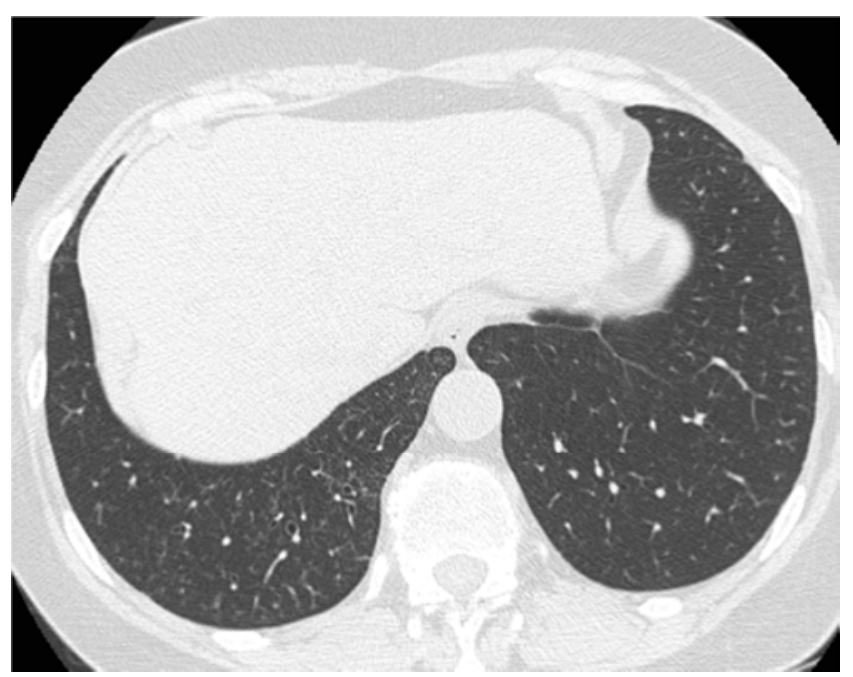

Figure 3. Particular CT: resolution of atelectasis.

\section{Discussion}

Despite the multiple drug therapy, our patient did not show any improvement. The patient with rheumatologic pathology may present with serious respiratory complications.

Water content of mucus affects viscosity and plays an important role in the transport of secretions. Physiologic humidification of secretions depends on proper functioning of airway epithelial cells. Breathing dry gas is desiccating, 
damaging epithelial cells and altering the characteristics of mucus. Warming inspired gas to the level of core temperature $\left(37^{\circ} \mathrm{C}\right)$ and humidifying it to saturation help to maintain adequate mucosal function and preserve the rheology and volume of secretions, maximizing mucociliary clearance without risk of thermal injury or over-humidification [2]. NHF is an option to simplify the removal and drainage of secretions in patients with bronchiectasis, due to its function of mucus hydration [3]. NHF also generates a positive end-expiratory pressure (PEEP), which may counterbalance auto-PEEP, further reducing work of breathing, improve oxygenation and stent airways open during expiration, permitting more complete emptying. Positive low-level airway pressure is also generated by NHF during inhalation, not depending on the closure of the mouth during breathing [4, 5]. Parke et al observed increase in tidal volume also in postcardiac surgery patients [6]. Tidal volume and end-expiratory lung volume increased the most in patients with a higher BMI. HFCWO generate either positive or negative trans-respiratory pressure excursions to produce high-frequency, small-volume oscillations in the airways. HFCWO can lead to changes in volume of $15-57 \mathrm{ml}$ and in flow up to $1.6 \mathrm{~L} / \mathrm{s}$, which generate minimal coughing to mobilize secretions. The typical treatment lasts 20-30 minutes, and consists of short periods of compression at different frequencies, separated by coughing. HFCWO has been shown to be effective in clearing secretions from the lungs of patients with bronchiectasis, asthma, cystic fibrosis, primary ciliary dyskinesia, emphysema, COPD [7] and in neuromuscular patient [8-10]. The combination of the rehabilitative methods allowed the total resolution of atelectasis in 3 weeks.

\section{Conclusion}

High flow nasal cannula (HFNC) supportive therapy has emerged as a safe, useful therapy for prespiratory patient and improving oxygenation and comfort. Recently several clinical trials have analyzed the effectiveness of HFNC therapy in different clinical situations and have reported promising results. Delivery of heated and humidified oxygen at high flow rates through nasal cannula is now widely used in adult patients. Its mechanisms of action and potential clinical benefits can help to improve the management of patients. COPD and bronchiectasis are both airway disorders characterized by neutrophilic airway inflammation, mucus hypersecretion and retention, and impaired mucociliary transport. In this patient, receiving HFNC therapy $\geq 2 \mathrm{~h}$ for day in their home, there have been excellent effects on the frequency of exacerbations, quality of life, lung function, exercise capacity, and airway inflammation. The combined use of High-Flow Therapy and High-Frequency Chest Wall
Oscillation to treath Persistent Pulmonary Atelectasis in Patient in patient with Rheumatoid Arthritis have been shown to be effective in clearing secretions from the lungs and for the complete resolution of the atelectasis area. Therefore, RCTs evaluating the efficiency of HFNC therapy in these situations are needed.

\section{Conflict of Interest}

The authors declare that they have no competing interests.

\section{References}

[1] T. Gono, H. Tokuda, F. Sakai, T. Takemura (Eds) (2018) Lung disease associated with rheumatoid arthritis. Springer Nature Singapore Pte Ltd., Singapore.

[2] Spoletini G, Alotaibi M, Blasi F, Hill NS, (2015). Heated Humidified High Flow Nasal Oxygen in Adults: Mechanisms of Action and Clinical Implications, Chest. Jul; 148 (1): 253-261.

[3] Hasani A, Chapman TH, McCool D, Smith RE, Dilworth JP, Agnew JE. (2008) Domiciliary humidification improves lung mucociliary clearance in patients with bronchiectasis. Chronic Respir Dis: 5 (2): 81-86.

[4] Groves N, Tobin A. (2007) High flow nasal oxygen generates positive airway pressure in adult volunteers. Aust Crit Care 20: 126-131.

[5] Braunlich J, Beyer D, Mai D, Hammerschmidt S, Seyfarth HJ, Wirtz H. (2012). Effects of nasal high flow on ventilation in volunteers, COPD and idiopathic pulmonary fibrosis patients. Respiration.

[6] Parke R, McGuinness S, Eccleston M. (2009). Nasal high-flow therapy delivers low level positive airway pressure. $\mathrm{Br} \mathrm{J}$ Anasesth. 103 (6): 886-890.

[7] Nicolini A., Grecchi B., Ferrari-Bravo M., Barlascini C. (2018) Safety and effectiveness of the high-frequency chest wall oscillation vs intrapulmonary percussive ventilation in patients with severe COPD. Int J Chron Obstruct Pulmon Dis. 16; 13: 617-625.

[8] Chakravorty I, Chahal K, Austin G (2011) A pilot study of the impact of high frequency chest wall oscillation in chronic obstructive pulmonary disease patients with mucus hypersecretion. Int J Chron Obstruct Pulmon Dis. 6: 693- 699.

[9] Chaisson KM, Walsh S, Simmons Z, Vender RL (2006) A clinical pilot study: high frequency chest wall oscillation airway clearance in patients with amyotrophic lateral sclerosis. Amyotroph Lateral Scler 7: 107-111.

[10] Lechtzin N., Wolfe LF, Frick KD (2016). The Impact of High-Frequency Chest Wall Oscillation on Healthcare Use in Patients with Neuromuscular Diseases. Ann Am Thorac Soc. 13 (6): 904-9. 